This item was submitted to Loughborough's Research Repository by the author.

Items in Figshare are protected by copyright, with all rights reserved, unless otherwise indicated.

\title{
Colossal magneto-optical conductivity in doped manganites
}

PLEASE CITE THE PUBLISHED VERSION

LICENCE

CC BY-NC-ND 4.0

\section{REPOSITORY RECORD}

Alexandrov, A.S., and A.M. Bratkovsky. 2019. "Colossal Magneto-optical Conductivity in Doped Manganites". figshare. https://hdl.handle.net/2134/1203. 


\title{
Colossal magnetooptical conductivity in doped manganites
}

\author{
A.S. Alexandrov ${ }^{1, *}$ and A.M. Bratkovsky ${ }^{2, \dagger}$ \\ ${ }^{1}$ Department of Physics, Loughborough University, Loughborough LE11 3TU, UK \\ ${ }^{2}$ Hewlett-Packard Laboratories, 3500 Deer Creek Road, Palo Alto, California 94304-1392
}

(March 10, 1999)

\begin{abstract}
We show that the current carrier density collapse in doped manganites, which results from bipolaron formation in the paramagnetic phase, leads to a colossal change of the optical conductivity in an external magnetic field at temperatures close to the ferromagnetic transition. As with the colossal magnetoresistance (CMR) itself, the corresponding magnetooptical effect is explained by the dissociation of localized bipolarons into mobile polarons owing to the exchange interaction with the localized Mn spins in the ferromagnetic phase. The effect is positive at low frequencies and negative in the high-frequency region. The present results agree with available experimental observations.
\end{abstract}

71.30.+h, 71.38.+i, 72.20.Jv, 78.20.Bh

As we have recently shown [1.2], the interplay of the electron-phonon and exchange interactions results in a current carrier density collapse (CCDC) at the paramagnetic-ferromagnetic transition in doped manganites. Owing to the strong electron-phonon interaction, polaronic carriers are bound into almost immobile bipolarons in the paramagnetic phase. A few thermally excited non-degenerate polarons polarize localized $\mathrm{Mn} d$ $\checkmark$ electrons. As a result, the exchange interaction breaks bipolarons below $T_{c}$ if the $p-d$ exchange energy $J_{p d} S$ of the polaronic carriers with the localized Mn $d$ electrons is larger than the bipolaron binding energy $\Delta$. Hence, the density of current carriers (polarons) suddenly increases below $T_{c}$, which explains the resistivity peak and CMR experimentally observed in many ferromagnetic oxides [3 5] as well as the giant isotope effect [6,7], the tunneling gap [8], the specific heat anomaly [9], along with the temperature dependence of the dc resistivity [10].

Another conspicuous general feature of the doped manganites is the massive spectral weight transfer in the optical conductivity to lower frequencies as the CMR materials are cooled below the Curie temperature [11 14. We have shown recently that this effect can be also understood as a result of CCDC 15. The high-temperature optical conductivity is well described by the bipolaron absorption, while the low temperature mid-infrared band is due to absorption by single polarons. A theoretical model in which the breaking of bipolarons caused by magnetic interactions below $T_{c}$ shifts the spectral weight from the bipolaronic peak to the polaronic one is in quantitative agreement with the optical spectra of the layered ferromagnetic $\left(T_{c}=125 \mathrm{~K}\right)$ crystals $\mathrm{La}_{2-2 x} \mathrm{Sr}_{1+2 x} \mathrm{Mn}_{2} \mathrm{O}_{7}$ 114. over the entire observed frequency and temperature range.

In the present paper we show that the optical properties of doped manganites should be extremely sensitive to the magnetic field (as suggested by the CMR itself). We predict an increase of the optical conductivity at low frequencies and a decrease at high frequencies with increasing magnetic field. The relative change of the optical conductivity is found to be very large near the phase transition.

The optical intraband conductivity of a charge-transfer doped insulator with (bi)polaronic carriers is the sum of the polaron $\sigma_{p}(\nu) \equiv e n \mu_{p}(\nu)$ and bipolaron $\sigma_{b}(\nu) \equiv e(x-$ $n) \mu_{b}(\nu)$ contributions at a given frequency $\nu$ 15. Their frequency dependence is described in the literature (for references see 19, 21,15]). The polaron and bipolaron optical mobilities have almost a Gaussian shape given by

$$
\mu_{p, b}(\nu)=\frac{\mu_{0} \mathcal{T}^{2}}{\nu \gamma_{p, b}} \exp \left[-\left(\nu-\nu_{p, b}\right)^{2} / \gamma_{p, b}^{2}\right]
$$

where $\mu_{0}=2 \pi^{1 / 2} e / a$ is a constant with $a$ the lattice spacing, $\mathcal{T}$ the hopping integral, $n=n(T, H)$ the (atomic) polaron density for a given temperature $T$ and magnetic field $H$, and $x$ the doping level. Here and below we assume $\hbar=c=1$. In general the polaron and bipolaron absorption have different maxima located around $\nu_{p}=4 E_{a}$ for polarons, and around $\nu_{b}=2 E_{p}-V_{c}$ for bipolarons [15], where $E_{a}$ is the dc activation energy, $E_{p}$ is the polaron (Franck-Condon) level shift and $V_{c}$ is the (inter-site) Coulomb repulsion. The broadenings of the polaronic, $\gamma_{p}$, and bipolaronic, $\gamma_{b}$, absorption are also different.

At temperatures above the ferromagnetic transition the polaron density is very low owing to CCDC [1] , so the intraband conductivity is due to bipolarons alone,

$$
\sigma(\nu)=\frac{e \mu_{0} x \mathcal{T}^{2}}{\nu \gamma_{b}} \exp \left[-\left(\nu-\nu_{b}\right)^{2} / \gamma_{b}^{2}\right]
$$

This expression fits the experiment 14 fairly well with $\nu_{b}=1.24 \mathrm{eV}$ and $\left.\gamma_{b}=0.6 \mathrm{eV} 15\right]$. When the temperature drops below $T_{c}$, at least some of the bipolarons are broken apart by the exchange interaction with $\mathrm{Mn}$ sites, because one of the spin-polarized polaron bands falls suddenly below the bipolaron level by an amount 
$\left(J_{p d} S-\Delta\right) / 2$ [目]. The intraband optical conductivity is determined now by both the polaronic and bipolaronic contributions, which explains the sudden spectral weight transfer from $\nu=\nu_{b}$ to $\nu=\nu_{p}$ observed below $T_{c}$ in the ferromagnetic manganites 12 14]. The experimental spectral shape in $\mathrm{La}_{2-2 x} \mathrm{Sr}_{1+2 x} \mathrm{Mn}_{2} \mathrm{O}_{7}$ at $T=10 \mathrm{~K}$ is well described with $n=x / 5, \nu_{p}=0.5 \mathrm{eV}$ and $\gamma_{p}=0.3$ $\mathrm{eV}$ [15.

The presence of a magnetic field changes the balance between polarons and bipolarons, resulting in a change of the optical conductivity. To quantify this magnetoptical effect we introduce the relative magnetoconductivity as

$$
\delta \sigma / \sigma \equiv[\sigma(\nu, H)-\sigma(\nu, 0)] / \sigma(\nu, 0) .
$$

Carriers in manganites are heavy. Hence, we do not expect any orbital effect of the magnetic field on the mobility. Only the carrier density is sensitive to the field in our theory. As a result we obtain

$$
\delta \sigma / \sigma=\frac{e[n(T, H)-n(T, 0)]\left[\mu_{p}(\nu)-\mu_{b}(\nu)\right]}{\sigma(\nu, 0)} .
$$

All major features of the magnetooptical conductivity can be understood with this expression. First of all the magnetooptical effect is maximal in the region of maximum variation of the polaron density $n$ with the external magnetic field. It follows from our equations (see below) that the effect is maximal close to $T_{c}$. The polaron mobility is larger than the bipolaron mobility at low frequencies and smaller at high frequencies, Eq.(1). At the same time one expects an increase of the polaron density with the magnetic field. Hence the magnetooptical effect should be positive in the low frequency region and negative at high-frequencies. To calculate the effect we use the Hartree-Fock equations [1,15] accounting also for the external magnetic field as

$$
\begin{aligned}
& n=\frac{t}{2 w} \ln \left[\frac{1+2 y \cosh \left(\frac{\sigma+h}{t}\right)+y^{2}}{1+2 y e^{-2 w / t} \cosh \left(\frac{\sigma+h}{t}\right)+y^{2} e^{-4 w / t}}\right], \\
& m=\frac{t}{2 w} \ln \left[\frac{1+2 y e^{-w / t} \cosh \left(\frac{\sigma+h+w}{t}\right)+y^{2} e^{-2 w / t}}{1+2 y e^{-w / t} \cosh \left(\frac{\sigma+h-w}{t}\right)+y^{2} e^{-2 w / t}}\right], \\
& \sigma=B_{2}[(4 h+m) /(2 t)],
\end{aligned}
$$

where

$$
y=e^{-\delta / t}\left[\frac{\sinh [(x-n) d /(2 x t)]}{\sinh [(x+n) d /(2 x t)]}\right]^{1 / 2} .
$$

Here $B_{S}(z)=[1+1 /(2 S)] \operatorname{coth}[(S+1 / 2) z]-$ $[1 /(2 S)] \operatorname{coth}(z / 2)$ is the Brillouin function, $m$ and $\sigma$ are the relative magnetization of polarons and of $\mathrm{Mn}$, respectively. The reduced temperature is $t=2 k_{B} T /\left(J_{p d} S\right)$, the dimensionless binding energy $\delta=\Delta /\left(J_{p d} S\right)$, and the dimensionless magnetic field is $2 \mu_{B} H / J_{p d} S$. These equations account for a finite polaron, $w=W / J_{p d} S$ and bipolaron, $d=D / J_{p d} S$, widths of the energy level distribution, essential at low temperatures. We also assume here that immobile bipolarons are localized by the impurities and there is no more than one bipolaron in a single localized state ('single well-single particle' approximation [22]). Therefore, the total number of states in the bipolaron (impurity) band is $x$.

The effect of magnetic field on optical conductivity is maximal near the transition temperature, where the system exhibits a massive spectral weight transfer between polaronic to bipolaronic peaks in optical conductivity. At low temperatures, $T<T_{c}$, when the system is almost polarized, $\sigma \approx 1$, the polaron density (5) depends on the field in the combination $1+\frac{h}{t} \tanh \left(\frac{\sigma}{t}\right)$. Since $h / t \ll 1$, the absolute change of the polaron density with the field in this region is always small. The same is true of the temperature range in the paramagnetic region far from the transition point. However, close to $T_{c}$ the effect is very large as follows from the main equations (5)-(8). Assuming a large carrier density collapse, $n_{c} / x \ll 1$ we readily obtain from Eq. (5) (with $\sigma=0$ ) the density of polarons at the critical temperature as

$$
n_{c}(h)=\frac{t_{c}(h)}{w} \exp \left[-\delta / t_{c}(h)\right]+O\left[\left(h / t_{c}\right)^{2}\right] .
$$

Here we assume that $w \ll t_{c}$ (in the opposite limit, $w \gg$ $t_{c}$, the preexponential factor will be 2 instead of $t_{c} / w$ ). It formally looks as if we have only a second-order effect of the field. However, $t_{c}=t_{c}(h)$ is very sensitive to the field [2] and this leads to a strong field dependence of the polaron density, and, consequently, to a colossal magnetooptical effect.

To illustrate the point, we have calculated $\delta \sigma / \sigma$ for $\delta=0.5\left(t_{c}=0.162\right)$, with parameters $\nu_{p, b}$ and $\gamma_{p, b}$ given above, assuming that $d \ll 1$ (Figs. 1, 2). With these parameters the phase transition is discontinuous, and it is accompanied by approximately an eight-fold decrease of the polaron density $n$ at the transition. Consequently, the low frequency (polaronic) peak at $\nu_{p}=0.5 \mathrm{eV}$ sharply reduces in height, as the spectral weight is suddenly transferred to the bipolaronic peak at $\nu_{b}=1.24 \mathrm{eV}$, for temperatures varying between $t=0.15$ and $t=0.175$, through the critical temperature, Fig. 11. With further increase in temperature the density of polarons increases again since the bipolarons are subject to thermal dissociation. As a result, we observe some re-entrant increase of the spectral weight of the polaronic peak at lower frequencies at $T>T_{c}$. It would be interesting to test this unusual temperature behavior of conductivity experimentally.

In a wide frequency interval we obtain a several-fold change in the magnetoconductivity calculated for $h=$ 0.005 and temperature $t=0.175$, which is somewhat higher than the critical temperature $t_{c}=0.162$.

The positive sign of the relative optical magnetoconductivity at frequencies, where the dominant contribution comes from the polarons, immediately follows from our picture of current carrier density collapse. Indeed, 


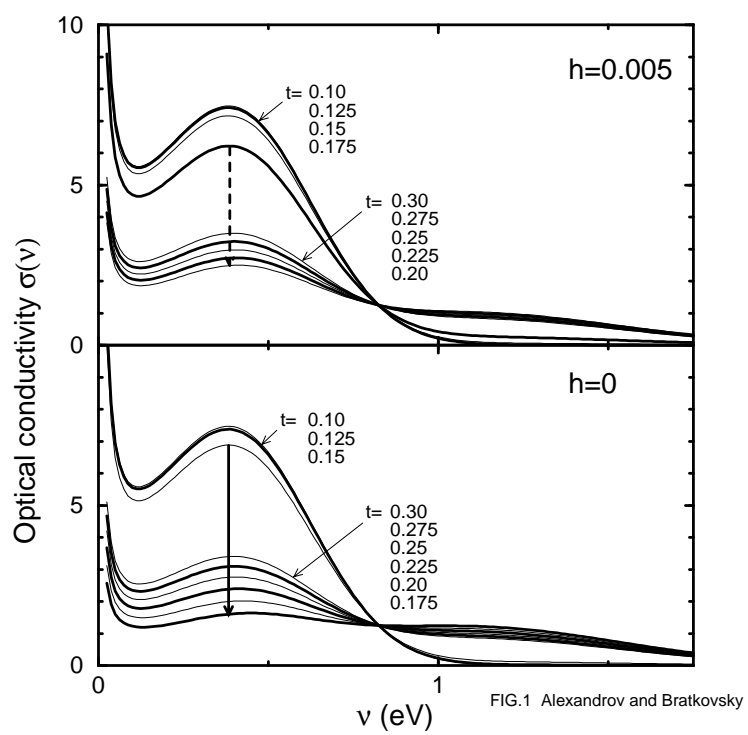

FIG. 1. Optical conductivity $\sigma(\nu)$ for various temperatures below and above the critical temperature $t_{c}=2 k_{B} T_{c} / J_{p d} S$ $\left(t_{c}=0.162\right.$ for $\left.\delta=\Delta / J_{p d} S=0.5\right)$. Background contribution to the absorption has been ignored [15]. The arrow indicates the sudden drop in the polaronic contribution to the conductivity around $\nu_{p}=0.5 \mathrm{eV}$ when the system is heated up through the critical temperature. Top panel: magnetic field $h \equiv 2 \mu_{B} H / J_{p d} S=0.005$; bottom panel: $h=0$. Note that the $\sigma(\nu)$ curve for $t=0.175$ raises from the bottom of the lower manifold of curves at $h=0$ to join the upper manifold of curves at $h=0.005$. For $\mathrm{La}_{0.75} \mathrm{Ca}_{0.25} \mathrm{MnO}_{3}$ we estimated $J_{p d} S=2250 \mathrm{~K}[1]$, so that the field $h=0.001$ corresponds to $H=1.67 \mathrm{~T}$.

the Zeeman splitting of the polaron band pulls one of the spin subbands down in energy. A fraction of the bipolarons, proportional to the magnetic field, will then be destabilized and dissociate into polarons. This is accompanied by an increase in the polaronic contribution to the conductivity and the positive sign of the magnetoconductance in the low frequency region. Below $T_{c}$ the relative changes in the conductivity are most pronounced in the region $\nu \geq 1.5 \mathrm{eV}$, where we have only tails of both polaron and bipolaron contributions. These changes are exaggerated because $\sigma(\nu, 0)$ itself is small in this region. Above $T_{c}$ the magnetooptical effect is mostly pronounced at low frequencies $\nu \leq 1 \mathrm{eV}$ (Fig. 2). Our theoretical prediction of a colossal and positive relative magnetoconductivity near $T_{c}$ is in agreement with recent measurements [16].

The spectral weight transfer is usually measured by the effective optical number of carriers

$$
N_{e f f}\left(\nu_{c}\right)=\frac{2 m_{0}}{\pi e^{2} N} \int_{0}^{\nu_{c}} d \nu \sigma(\nu)
$$

where $m_{0}$ is the bare electron mass, $N$ is the number of, for instance, dopant atoms per unit volume, $\nu_{c}$ is the cutoff energy, which spans a low-frequency region (e.g. $\nu_{c}=0.5 \mathrm{eV}$ [13], $0.8 \mathrm{eV}$ 14]). According to the sum rule

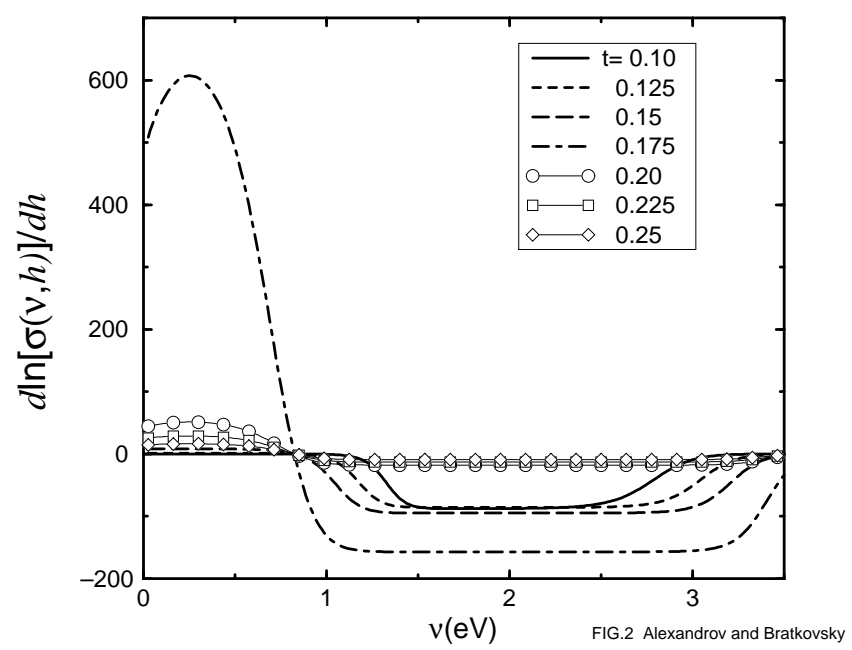

FIG. 2. Differential relative magnetoconductivity for the system with the same parameters as in Fig. 1. Note the huge change in the conductivity close to the critical temperature $t_{c}=2 k_{B} T_{c} / J_{p d} S=0.162$. In a field $H=16 \mathrm{~T}(h \approx 0.01)$, as in experiment [16], the maximal effect is $(\delta \sigma / \sigma)_{\max }=5.7$ at $\nu=0.25 \mathrm{eV}$ and $t=0.175$ (close to $T_{c}$ ).

for conductivity, $N_{\text {eff }}(\infty)$ equals the number of carriers in the system. By using a finite-frequency cutoff, one can estimate the integral weight transfer from a given interval of energies. Typical behavior of doped manganites is that $N_{e f f}$ for mid-IR gradually diminishes with elevated temperature in the ferromagnetic phase until the systems approaches a critical temperature, where the weight drops. With the mobilities, Eq. (1), calculated within a (nonconserving) saddle-point approximation one is unable to quantify the integral spectral weight transfer. However, its experimental behavior is in qualitative agreement with our notion of the current carrier density collapse as described above.

In conclusion, we have developed the theory of the magnetooptical conductivity in doped magnetic chargetransfer insulators with a strong electron-phonon interaction. We have found a colossal magnetooptical effect close to the critical temperature, positive at low frequencies and negative at high frequencies. The conductivity increase in the field is several-fold at low frequencies near the phase transition in accordance with that observed experimentally 16. Our theory of doped manganites is largely independent of the symmetry of the relevant electron orbitals and of the type of electron-phonon interaction. Its key feature is the pairing of carriers in the paramagnetic phase due to the lattice distortion and their unpairing in the ferromagnetic phase due to the exchange pair-breaking interaction, the latter being reminiscent of the magnetic pair-breaking in superconductors with magnetic impurities. It would be interesting to check the predicted strong temperature dependence of the magne- 
toconductivity experimentally on various CMR systems.

We would like to acknowledge useful discussions with D.N. Basov pertaining to peculiarities of optical properties of strongly correlated systems. We are grateful to G. Aeppli, A.R. Bishop, D.S. Dessau, M.F. Hundley, K.M. Krishnan, A.P. Ramirez, R.S. Williams, and Guo-meng Zhao for helpful discussions. ASA acknowledges support from the Quantum Structures Research Initiative and the External Research Program of Hewlett-Packard Laboratories (Palo Alto).

* $\quad$ Email asa21@cus.cam.ac.uk

$\dagger \quad$ Email alexb@hpl.hp.com

[1] A.S. Alexandrov and A.M. Bratkovsky, Phys. Rev. Lett. 82, 141 (1999).

[2] A.S. Alexandrov and A.M. Bratkovsky, J. Phys. Condens. Matter 11, 1989 (1999).

[3] G.H. Jonker and J.H. Van Santen, Physica (Utrecht) 16, 337 (1950); J.H. Van Santen and G.H. Jonker, Physica (Utrecht) 16, 599 (1950).

[4] R. von Helmolt et al., Phys. Rev. Lett. 71, 2331 (1993).

[5] S. Jin et al., Science 264, 413 (1994).

[6] Guo-meng Zhao et al., Nature 381, 676 (1996).

[7] J.P. Franck et al., Phys. Rev. B 58, 5189 (1998).

[8] A. Biswas et al., cond-mat/9806084.

[9] A.P. Ramirez, J. Phys. Condens. Matter 9, 8171 (1997).

[10] P. Schiffer et al., Phys. Rev. Lett. 75, 3336 (1995).

[11] Y.Okimoto et al., Phys. Rev. Lett. 75, 109 (1995).

[12] Y.Okimoto et al., Phys. Rev. B55, 4206 (1997).

[13] K.H. Kim, J.H. Yung, and T.W. Noh, Phys. Rev. Lett. 81, 1517 (1998).

[14] T. Ishikawa et al., Phys. Rev. B 57, R8079 (1998).

[15] A.S.Alexandrov and A.M.Bratkovsky, cond-mat/9901340 (1999).

[16] A.V. Boris et al., Phys. Rev. B 59, 697 (1999).

[17] D.S. Dessau et al., Phys. Rev. Lett. 81, 192 (1998).

[18] J.-S. Zhou et al., Phys. Rev. Lett. 79, 3234 (1997).

[19] H. Böttger and V.V. Bryksin, Hopping Conduction in Solids (Academic-Verlag, Berlin, 1985).

[20] G.D. Mahan, Many-Particle Physics (Plenum, New York, 1990).

[21] A.S. Alexandrov and N.F. Mott, Polarons and Bipolarons (World Scientific, Singapore, 1995).

[22] A.S. Alexandrov, A.M. Bratkovsky, and N.F. Mott, Phys. Rev. Lett. 72, 1734 (1994). 